DOI: https://doi.org/10.46296/gt.v4i8edesp.0045

\title{
DESINTEGRACIÓN FAMILIAR Y DESARROLLO DE LOS NIÑOS EN LA PRIMERA INFANCIA
}

\section{FAMILY DESINTEGRATION AND DEVELOPMENT OF CHILDREN IN EARLY CHILDHOOD}

\author{
Quijano-Murgueytio Josselyn Andrea ${ }^{1}$; Quiroz-Rivadeneira Carmen Monserrate ${ }^{2}$ \\ ${ }^{1}$ Maestría Académica con Trayectoria de Investigación en Psicología, Mención Psicoterapia. \\ Instituto de Posgrado, Universidad Técnica de Manabí, UTM. Portoviejo, Ecuador. Correo: \\ jquijano1314@utm.edu.ec. ORCID ID: https://orcid.org/0000-0001-9547-0221 \\ ${ }^{2}$ Instituto de Posgrado, Universidad Técnica de Manabí, UTM. Portoviejo, Ecuador. Correo: \\ carmen.quiroz@utm.edu.ec. ORCID ID: https://orcid.org/0000-0002-9805-2065
}

\begin{abstract}
Resumen
En esta investigación, se aborda la desintegración familiar, problema que siempre ha estado presente y en la actualidad va en aumento dentro de los hogares ecuatorianos. El principal objetivo de este estudio es analizar los factores que conllevan a desintegrar una familia y cómo esto repercute durante el desarrollo integral de los niños en la primera infancia. Este trabajo es de diseño no experimental, cuali-cuantitativo, de corte transversal y de tipo explicativo. Como método empírico se aplicó el test APGAR familiar a padres de familia de la Unidad Educativa Fiscal en la ciudad de Portoviejo, para detectar la funcionalidad e integración familiar dentro de estos sistemas. En los resultados, se encuentra que las familias funcionales e integradas predominan en este estudio, lo cual refleja que existe buena comunicación entre sus integrantes, apoyo emocional y fortaleza ante un problema. En conclusión, la familia es la base fundamental para el crecimiento y desarrollo de los niños en todos sus aspectos.
\end{abstract}

Palabras clave: Desintegración familiar, integración familiar, desarrollo infantil, primera infancia.

\begin{abstract}
In this research, is about the family's disintegration, it is a problem that has always existed and is currently increasing in Ecuadorian households. The main objective of this study is to analyze the factors that lead to disintegration of a family and how this affects the integral development of children in early childhood. This work is of a non-experimental, quali-quantitative, cross-sectional and explanatory type design. As an empirical method, the family APGAR test was applied to parents of the Fiscal Education Unit in the city of Portoviejo, to detect the functionality and family integration within these systems. In the results, it is found that functional and integrated families predominate in this study, which reflects that there is good communication between their members, emotional support, and strength in the face of a problem. In conclusion, the family is the fundamental basis for the growth and development of children in all its aspects.
\end{abstract}

Keywords: Family desintegration, integration family, child development, early childhood.

Información del manuscrito:

Fecha de recepción: 13 de julio de 2021.

Fecha de aceptación: 18 de octubre de 2021.

Fecha de publicación: 12 de noviembre de 2021. 


\section{Introducción}

La desintegración familiar es una problemática que radica en muchas familias por diversas razones. En varios países latinoamericanos se ha experimentado un número significativo de cambios en la vida familiar en las últimas décadas, debido a aspectos como incremento de madres solteras y mujeres como jefas de hogar, esto asociado con los niveles decrecientes de matrimonios, el aumento de nacimientos fuera de matrimonio, tasas altas de divorcio, separación, entre otros (Intriago, 2020).

La desintegración familiar se muestra como la ruptura de la unión que se tiene como miembros de un mismo sistema. Esta situación trae como consecuencias la afectación de cada uno de sus integrantes de manera individual, puede ser emocional o psicológica, económica, académica o laboral.

Con relación al caso de la Unidad Educativa Fiscal Juan Montalvo, ubicada en la parroquia San Pablo de la ciudad de Portoviejo, provincia de Manabí Ecuador, institución que de acuerdo con datos del Departamento de Consejería Estudiantil, existen estudiantes que viven dentro de esta situación y se encarga de hacer un seguimiento de los casos para brindar la ayuda correspondiente.

Un estudio de Zapata (1999), citado por Norka, 2013 refiere la desintegración familiar como conflictos irresueltos que generan descomposición en las relaciones familiares que les aleja de los proyectos comunes para cumplir el rol como núcleo de la sociedad en un marco de armonía y bienestar que se proyecte al desarrollo personal de cada miembro del grupo familiar.

Un hogar unido, conformado e integrado por una familia en donde la base de esta sean los valores, principios, y la comunicación, influenciará de manera positiva en el desarrollo emocional y en el crecimiento del niño como ser humano, lo cual es indispensable para la formación de su personalidad.

Es fundamental tener en cuenta el impacto que tiene la ruptura familiar en los hijos, lo cual puede varía según la edad y va seguido de una serie de cambios significativos que conllevan esfuerzo para adaptarse a la nueva realidad, lo que hace necesario como profesionales 
apoyar y acompañar a las familias en este proceso.

La familia es la unión base de una sociedad, las cuales están conformadas por madre, padre e hijos unidos por los lazos de parentesco o vinculo consanguíneo. Existen diferentes tipos de familias como las nucleares, monoparentales, mixtas, extensas, entre otras.

Según (Girondas, 2016) La familia es el medio que transmite a sus hijos, valores, principios y sentimientos que a través de una buena comunicación generan confianza y positivismo para desarrollarse dentro de su entorno; o por el contrario pueden transmitir rechazo, desconfianza e inseguridades, esto depende del ambiente en el que vive este sistema.

La primera infancia es fundamental para el desarrollo integral de los niños. Por medio de las experiencias, emociones y sucesos se va construyendo su propia identidad y la formación de su personalidad. Esta primera etapa que va desde cero a cinco años, es decisiva en el desarrollo, pues de ella va a depender toda la evolución posterior del niño en aspectos motor, lenguaje, cognitivo y socio afectivo.

De acuerdo con (Bandura, 1987) Cada persona adquiere conocimiento a través de la observación de la conducta de los demás, una vez adquirida nueva información, esta se replicará del modelo observado a través de acciones y nos permitirá tener nuestra propia experiencia.

Para (Silva, 2015) el contacto del niño con el mundo en la etapa inicial es fundamental, no solo para su desarrollo físico motor, sino también para la formación de su personalidad, la cual por medio de vivencias fortalecerán sus cualidades y actitudes que le facilitan la relación con el ambiente y su alrededor.

La interacción del niño dentro de un espacio social es favorecedor para su desarrollo psíquico y emocional, pues esto le permite ser parte de una identidad cultural que da sentido a su existencia y a la de su familia, la cual les admite tener costumbres y tradiciones lo que genera un impacto como ser humano (Castillo, 2017).

Según (Posada, 2014) formar niños dentro de un sistema estable, 
afectuoso, armonioso, en donde se sienten apoyados, motivados $\mathrm{y}$ protegidos hará que un futuro quieran buscar lo mismo y de esa forma ofrecerlo a los demás.

El rol de la familia en la primera infancia es primordial para el desarrollo cognitivo, emocional y social, se brinda protección, cuidado y educación para fortalecer su progreso, sin embargo hay hogares en donde existe violencia intrafamiliar que afecta al entorno familiar como tal.

La familia es la principal fuente para el desarrollo integral de los niños, en esta etapa se los educa en diferentes ámbitos, lo cual les permitirá desenvolverse en su entorno e ir fortaleciendo su identidad, cada familia tiene su propia manera de relacionarse y comunicarse donde se diferencian unas de otras. (Marín, 2018).

(Romero, 2019) Afirma que uno de mayores problemas que existen en muchos países es la desintegración familiar, la que genera una ruptura del sistema familiar, lo cual afecta su funcionamiento y produce un desequilibrio en el núcleo.
(Vargas, 2017) Manifiesta que la familia es quien se encarga de cubrir las necesidades de supervivencia básicas de cada uno de sus miembros, como: vestimenta, medicina, alimentación, vivienda, educación y entre otras. Así mismo son importantes las necesidades afectivas como: amor, protección, interés, comprensión, cariño y respeto.

Por esta razón, el interés por desarrollar este estudio tiene como objetivo determinar la influencia que tiene la desintegración familiar en el desarrollo de los niños durante la primera infancia, debido a que ellos son los más perjudicados al desarrollarse dentro de sistemas divididos, lo que implica efectos negativos en este periodo.

\section{Metodología}

Esta investigación es de diseño no experimental, cuali-cuantitativo, de corte transversal y de tipo explicativo. Se utilizó como técnica de recolección de información, entrevistas a tres profesionales en psicología dentro del área infantil y familiar, así mismo a la directora de la Unidad Educativa Fiscal Juan Montalvo de Portoviejo, para 
conocer datos de la institución, el número de estudiantes que están dentro del rango de edad de la primera infancia, y los contextos familiares. Según su reporte, hay 30 niños en Primer año de Educación Básica, los cuales son la población a investigar. Al ser una cantidad pequeña, se trabajó con la totalidad. Como método empírico se aplicó el Test APGAR Familiar a los padres de familia o representantes de los estudiantes.

Este instrumento muestra cómo perciben los miembros de la familia el nivel de funcionamiento e integración de la unidad familiar de forma global. Las preguntas valoran el contexto familiar en las áreas de adaptación, vida en común, crecimiento, afecto y resolución de conflictos. Posteriormente se obtiene información que proporciona datos básicos sobre el nivel de dinámica familiar, dando una idea de qué áreas necesitan una valoración e intervención profesional más detallada y de las redes y recursos familiares que pueden utilizarse para solucionar diversos conflictos.

El APGAR se califica de la siguiente manera: 0 nunca, 1 casi nunca, 2 algunas veces, 3 casi siempre y 4 siempre. Las puntuaciones de cada una de las cinco preguntas se suman posteriormente. No sólo sirve para detectar la disfuncionalidad familiar sino que guía al profesional a explorar aquellas áreas que la persona ha señalado como problemáticas.

\section{Resultados}

De acuerdo con el autor del test (Gabriel Smilkstein, 1978) en las puntuaciones existen cinco parámetros que al sumarlos el puntaje fluctúa entre 0 y 20 , lo que indica una baja, mediana o alta satisfacción en el funcionamiento de la familia.

Los padres de familia 0 representantes de los niños que realizaron el test fueron 30 , luego de la calificación del instrumento se obtuvo el porcentaje mayor en un $47 \%$ que corresponde a la categoría de familia funcional, lo que indica que la unión familiar prevalece dentro del entorno; un 33\% que pertenece a moderadamente disfuncional; es decir familias que empiezan con dificultades dentro del sistema que pudieran bajar a la siguiente categoría, grave disfunción que tiene un $20 \%$, esto muestra 
familias en situaciones críticas en

desintegrado o está próximo a donde la unidad familiar se ha hacerlo.

Tabla 1. Niveles de funcionalidad familiar en los hogares de Primer Año de Educación Básica de la Unidad Educativa Juan Montalvo.

\begin{tabular}{|c|c|c|}
\hline Nivel de funcionalidad & $\mathbf{F}$ & $\%$ \\
\hline Familia funcional & 14 & 47 \\
\hline Moderadamente disfuncional & 10 & 33 \\
\hline Grave disfunción & 6 & 20 \\
\hline Total & 30 & 100 \\
\hline
\end{tabular}

Fuente: Autores (2021).

La siguiente información fue obtenida a través de entrevista realizada a tres profesionales en el área de psicología, los cuales laboran en instituciones educativas fiscales, particulares y fundaciones de atención, en donde se encuentran a diario con diversas situaciones de familias.

Tabla 2. Factores de la desintegración familiar.

¿Cuáles son las causas que llevan a desintegrar una familia?

¿De qué manera afecta la desintegración del
Psicóloga 1: El maltrato físico/psicológico, el divorcio, el abandono de alguno de los padres, la falta de comunicación entre los miembros de la familia.

Psicóloga 2: El consumo de sustancias psicotrópicas, la religión, la violencia familiar, el abandono de uno de los conyugues, el divorcio o disolución legal de un matrimonio, la muerte de un miembro o de ambos, la migración, la comunicación inadecuada, carencias afectivas, el desempeño inadecuado de sus obligaciones o deberes en el rol correspondiente, la pérdida de objetivos comunes, distanciamiento físico y psíquico, entre otros.

Psicólogo 3: Divorcios, drogodependencia por uno de los padres, violencia física o psicológica y pobreza extrema.

Psicóloga 1: No permite su concentración y al verse afectado es difícil que logre desarrollar sus capacidades.

Psicóloga 2: Esto provoca problemas emocional y afectivo, que se interponen en su desempeño académico, su autoestima tiende a bajar, le ocasiona bajo rendimiento escolar y a veces deserción, también existen la posibilidad de alteraciones de las emociones básicas y problemas de 


\begin{abstract}
hogar de un niño en su desarrollo?

las relaciones interpersonales dentro y fuera de su entorno académico o familiar.

Psicólogo 3: Afecta directamente el desarrollo socioemocional porque puede generar autoestima baja, inseguridad, aislamiento, sentimientos de abandono y de inferioridad que pueden aparecer o mantenerse hasta la vida adulta, también experimentan eventos traumáticos, puesto que sus padres no les brindan la atención, confianza y apoyo que requieren.
\end{abstract}

Psicóloga 1: Buena comunicación entre todos los miembros de la familia, buen trato y compartir actividades entre todos.

¿Qué aspectos son importantes dentro una familia para que esta sea funcional?
Psicóloga 2: Una familia equilibrada y estructurada favorece las funciones de todos sus miembros, pendiente siempre por las necesidades básicas alimentos, vestimenta , vivienda, compromiso. Esto favorece la salud mental y fortalece la personalidad cubriendo las necesidades afectivas. La buena funcionalidad requiere que sea una familia multidireccional, donde todos los integrantes influyen entre sí.

Psicólogo 3: Promover un desarrollo favorable a la salud para todos sus miembros, para lo cual es imprescindible que tenga: jerarquías, límites, roles claros y definidos, comunicación abierta y explícita y capacidad de adaptación al cambio.

Fuente: Autores (2021).

Resultado de esta investigación se encuentra que las familias funcionales e integradas predominan, lo cual refleja que existe buena comunicación entre sus integrantes, apoyo emocional, fortaleza ante un problema, afectividad, interés por las actividades de los otros y tiempo de calidad que comparten como familia. Sin embargo sumando los porcentajes de los niveles moderado y gravemente disfuncional, este supera el valor mayor del nivel de familia funcional, es decir, que en general, estos entornos familiares se encuentran divididos casi en mitad funcionales y la otra mitad con problemas dentro de su sistema como falta de comunicación, carencias afectivas, falta de interés por los demás miembros, no respetar los límites y roles dentro de la convivencia y dificultades económicas. 
Cabe mencionar que es fundamental que los niños durante el periodo de la primera infancia, se desarrollen en un ambiente familiar lleno de valores, principios, normas y límites que le permitirán ser disciplinado, cuidadoso y responsable con sus actividades. Es en esta etapa en donde los niños van absorbiendo todo lo que está en su entorno, tanto familiar como social, para hacerlos parte de la construcción de su personalidad y posteriormente de su identidad como niño o niña y en un futuro estas serán las bases de su comportamiento como adulto.

\section{Discusión}

En un estudio realizado en Bogotá con adolescentes de acuerdo con lo hallado en el APGAR Familiar, el $39.7 \%$ tienen un núcleo familiar funcional, el $44.4 \%$ presenta disfuncionalidad moderada y finalmente el $15.9 \%$ presenta disfuncionalidad severa (Moreno \& Echeverría, 2014). A diferencia de nuestros resultados, en estos valores prevalecen las familias con una disfuncionalidad modera da, esto podría ser porque los contextos sociales de estos dos países son diferentes o porque en uno se trabaja con niños y en el otro con adolescentes

A partir de lo observado en otras investigaciones realizada por (Alvarez, Ayala, Nuño, \& Alatorre, 2005) la prevalencia se muestra en el $82.5 \%$ de las familias que tuvieron un puntaje global funcional, y sólo el $17.5 \%$ obtuvo un valor de puntaje global por abajo del valor límite funcional.

Los resultados antes descritos, aportan a que la composición e integración familiar dependen de los valores, normas y responsabilidades que practican como miembros de la misma y de la función que cumple cada uno, por esta razón muchas veces no se otorga la importancia que tiene la familia en el desarrollo de los niños durante los primeros años de vida, por eso se observa deficiencias en el progreso de los infantes tanto de manera personal como social que llevan a influir en la parte psicológica emocional del niño y su entorno. 


\section{Conclusiones}

Los resultados de la investigación indican que un mayor porcentaje de los niños de primer año de educación básica de la Unidad Educativa Juan Montalvo viven en un entorno familiar funcional, lo que aporta efectivamente en la formación de la personalidad y en su desarrollo integral.

Los principales factores que conllevan a desintegrar una familia son falta de comunicación y valores, el divorcio, problemas económicos y violencia intrafamiliar.

Los profesionales de psicología recomiendan para el desarrollo y bienestar infantil, que dentro del entorno familiar, las relaciones entre sus miembros sea integradora para tener mejor convivencia y fortalecer el mismo.

\section{Bibliografía}

Álvarez, A., Ayala, A., Nuño, A., \& Alatorre, M. (2005). Estudio sobre el nivel de funcionalidad en un grupo de familias que tienen un hijo con parálisis cerebral infantil. Revista Mexicana de Medicina Física y Rehabilitación.

Castillo, S. (2017). La desintegración familiar: Impacto en el desarrollo emocional de los niños. Revista ciencia e investigación, VOL. 2, NO. 9.

Girondas, R. (2016). Efectos de la desintegración familiar en el rendimiento académico de los estudiantes. Perú.

Intriago, A. (2020). Influencia de la desintengración familiar en el desarrollo escolar. Revista Científica y Arbitrada de Ciencias Sociales y Trabajo Social "Tejedora" Vol.3.

Marín, M. (2018). Influencia de las relaciones familiares en la primera infancia. Poiésis, (36), 164-183.

Moreno, J., \& Echeverría, K. (2014). Funcionalidad familiar, conductas internalizadas y rendimiento académico en un grupo de adolescentes. Psychologia: avances de la disciplina, 8(2), 37-46.

Norka, O. (2013). Causas de la desintegración familiar y sus consecuencias en el rendimiento escolar. Perú.

Posada, F. Q. (2014). Método de evaluación

para intervenciones en apego dirigidas a primera infancia. Revista Latinoamericana de Ciencias Sociales, Niñez y Juventud, 13 (2), pp. 10511063.

Romero, S. (2019). Desintegración familiar y rendimiento académico.

Revista 
Contribuciones a las Ciencias Sociales.

Silva, R. D. (2015). La actividad infantil y el desarrollo emocional en la infancia. Revista Intercontinental de Psicología y Educación, vol. 16., pp. 9-30.

Vargas, M. (2017). La desintegración familiar y el aprendizaje. Revista Ciencia e Interculturalidad.

Vélez, L. D. C., Gutiérrez, L. F. G., \& Gómez, V. G. (2018). El papel del psicólogo en la protección de la primera infancia frente a los fenómenos de violencia en Colombia. Poiésis, 1(34), 152165. 